\title{
Barreras y facilitadores para el uso de preparados de base alcohólica: Elemento clave de la estrategia multimodal para aumentar la adherencia a la higiene de manos. Revisión sistemática cualitativa
}

\author{
Barriers and motivating factors in the use of alcohol based products: The key to a \\ multimodal strategy to increase compliance with hand hygiene practices. \\ Qualitative systematic review.
}

\author{
Karen Ulloa Catalán $n^{1,2}$
}

'Programa de Doctorado en Enfermería, Facultad de Enfermería Universidad Andrés Bello (Santiago, Chile).

2Programa Control de Infecciones, Hospital de Urgencia Asistencia Pública, Santiago, Chile.

Financiamiento: Sin financiamiento

Conflicto de intereses: Autora declara no tener conflicto de intereses.

Recibido: 21 de noviembre de 2019 / Aceptado: 12 de diciembre de 2020

\section{Resumen}

Introducción: La adherencia a la higiene de manos (HM) por parte del personal de salud es de $38 \%$ a nivel mundial. Con la estrategia multimodal de la OMS se incluyeron los preparados de base alcohólica como un componente para la mejora de la HM. La campaña "los 5 momentos de la HM" incentiva a utilizar este producto que, entre otros beneficios, su aplicación ocupa menor tiempo en comparación con el lavado de manos. Objetivo: Conocer cuáles son los factores facilitadores o las barreras que favorecen o dificultan el uso de preparados de base alcohólica por el personal de salud para aumentar la adherencia a la HM según la estrategia multimodal de la OMS. Métodos: Fueron utilizadas dos bases de datos PubMed y CINHAL (años 2009 - 2019). Los artículos fueron seleccionados según criterios de inclusión - exclusión. Resultados: De 30 artículos se seleccionaron 12 , los que tenían como tema central la adherencia a la HM, personal de salud y preparados de base alcohólica. Se organizaron en facilitadores y barreras: infraestructura, presentación del producto y capacitación del personal. Discusión: Los facilitadores y barreras que más impacto reportan en aumentar la adherencia a la HM son la infraestructura, acceso, disponibilidad en el punto de atención, presentación del producto y capacitación al personal de salud sobre los productos de base alcohólica para la desinfección de las manos, con el fin de disminuir las IAAS y brindar una atención segura.

Palabra clave: higiene de manos; sanitizantes de manos; control de infecciones; preparados de base alcohólica.

\section{Abstract}

Introduction: The compliance with $\mathrm{HH}$ (hand hygiene) practices by health personnel is $38.7 \%$ worldwide. Using a multimodal strategy of the WHO (World Health Organization), alcohol based products was introduced. The campaign "The five moments of $\mathrm{HH}$ " encourages the use of this product which offers countless benefits, an important one being that it takes much less time compared to hand washing. Objecti$v e$ : To discover what the motivating factors or barriers are that favor or hinder the use of alcohol based products by health personnel, in order to increase compliance with $\mathrm{HH}$ according to the WHO multimodal strategy. Methods: Two databases were used; PubMed and CINHAL (from 2009 - 2019) The articles were selected according to inclusion - exclusion criteria. Results: From 30 articles, 12 were selected that had HH compliance, health personnel and alcohol based products as their main topics. They were organized into motivating factors and barriers: using infrastructure, product presentation and staff training. Discussion: Discovering the motivating factors and the barriers is essential to directing the efforts of strategies that include these points and to increase the compliance of $\mathrm{HH}$ with health personnel in order to reduce the HCAI (Health Care Associated Infection) and provide safe healthcare.

Keywords: Hand hygiene; hand sanitizers; infection control; alcohol based products. 


\section{Introducción}

\section{$\mathrm{L}$}

a higiene de manos (HM) corresponde a la principal medida de prevención contra las infecciones asociadas a la atención de salud (IAAS) ${ }^{1}$. Forma parte del primer desafío mundial de la seguridad del paciente: "una atención limpia es una atención segura", año $2005^{2}$. La importancia de la HM para prevenir las IAAS es ampliamente reconocida por el personal de salud, sin embargo, la adherencia a esta práctica en todo el mundo es baja, fluctuando entre 5 y $89 \%$, con un promedio de $38 \%{ }^{1}$. Las manos son cruciales en la diseminación de agentes multi-resistentes. Se estima que entre 20 y $40 \%$ del total de las infecciones cruzadas derivan del contacto con el paciente y su entorno ${ }^{3}$. Una vez que los microorganismos multi-resistentes colonizan las manos del personal pueden sobrevivir varios minutos, aumentando el riesgo de transmisión hacia los pacientes o al entorno hospitalario ${ }^{4}$. Como consecuencia de esta transmisión, aumenta la probabilidad de adquirir una IAAS, lo que impacta en una mayor morbi-mortalidad de los pacientes ${ }^{5}$ y en el aumento de los costos sanitarios, reflejado en el aumento de los días cama y uso de antimicrobianos. Para el paciente y su familia trae consigo una serie de efectos perjudiciales como: ausentismo laboral, gastos económicos, problemas psicológicos, entre otros ${ }^{6}$.

Aumentar la adherencia a la HM se traduce en una disminución de las IAAS $^{4}$ y consecuentemente, en el impacto que estas provocan en el paciente, familia y servicios de salud.

El objetivo de esta revisión es revisar cuáles son los factores facilitadores o las barreras que favorecen $o$ dificultan el uso de preparados de base alcohólica por el personal de salud con el fin de aumentar la adherencia a la HM, según la estrategia multimodal de la OMS.

\section{Metodología}

Revisión sistemática cualitativa. Se realizó una búsqueda de artículos, publicados dentro de los años 2009-2019 en las bases de datos PubMed y CINHAL, con el propósito de revisar las publicaciones relacionadas a los facilitadores y/o barreras para la adherencia del personal de salud a la HM tras la implementación del uso de preparados de base alcohólica.

Los criterios de inclusión en la selección de los artículos fueron: estudios observacionales, descriptivos, de intervención, cualitativos y cuantitativos que describieran los facilitadores y/o barreras para el uso del alcohol gel en el personal de salud; artículos publicados en idioma inglés, español; últimos 10 años de antigüedad, tomando como hito el lanzamiento de la estrategia multimodal de la OMS.
Los criterios de exclusión fueron: acceso solo a resúmenes; artículos que no tuvieran como tema el alcohol gel o preparados de base alcohólica; revisiones sistemáticas tipo metaanálisis.

La estrategia de búsqueda en las bases de datos mencionadas utilizó los siguientes descriptores: ("Hand Hygiene"[Mesh] AND "Hand Sanitizers"[Mesh]) AND "Health Personnel"[Mesh]. Se ocuparon los recursos de información bibliográfica de la Universidad Nacional Andrés Bello.

Se realizó el análisis de contenido de los artículos seleccionados tomando la sección de Métodos, Resultados y Conclusiones.

De los artículos de investigación se seleccionaron aquellos términos que se repetían en relación al uso del alcohol gel como estándar de oro en la estrategia multimodal de la OMS.

\section{Resultados}

La búsqueda inicial encontró 56 artículos con título relacionado con HM y uso de preparados de base alcohólica o alcohol gel. Tras el análisis de los títulos y resúmenes se redujo la selección a 30 artículos, los que cumplían con los criterios de inclusión y exclusión. Luego de realizar un análisis del texto completo, se excluyeron 18 por no desarrollar el tema central de la revisión (Figura 1).

Los artículos incluidos en esta revisión fueron de tipo: observacionales $(n=4)$, cualitativos $(n=2)$ y cuasi-experimentales $(n=6)$. Los artículos seleccionados se agruparon en: facilitadores y barreras para aumentar la adherencia a la HM con el uso de preparados de base alcohólica. Cada grupo hacía referencia a la infraestructura, capacitación

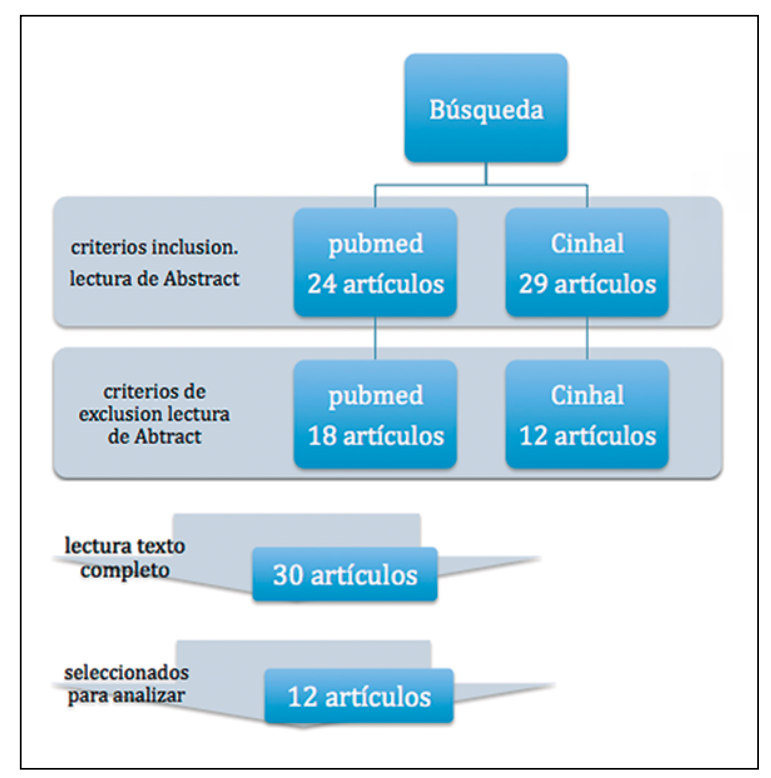

Figura 1. Resultados de la búsqueda bibliográfica sobre facilitadores y/o barreras en el uso de preparados de base alcohólica en la higiene de manos. 
Figura 2. Los resultados presentados como facilitadores y barreras del uso del alcohol gel.

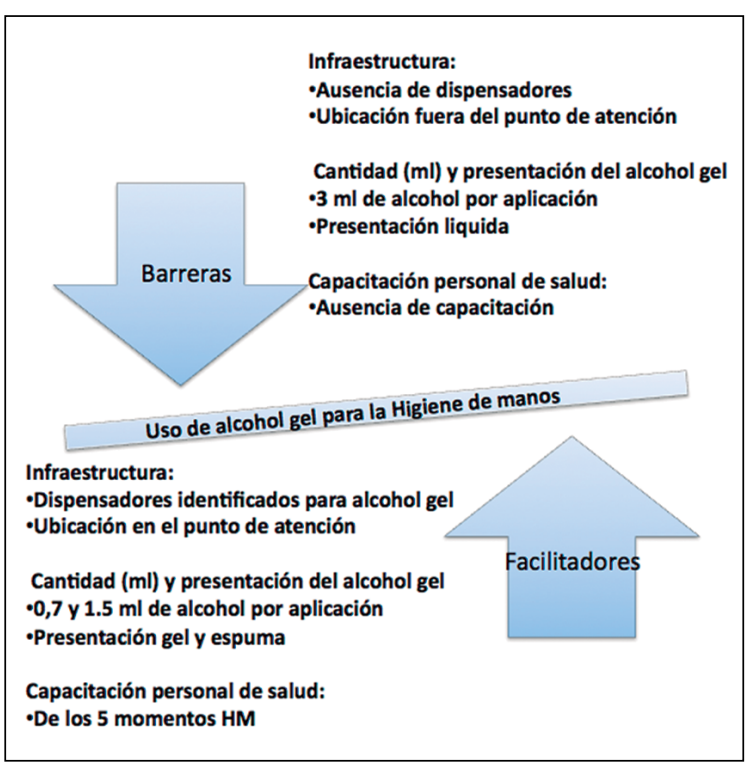

del personal de salud, cantidad (ml) según tolerabilidad y presentación del producto a utilizar (líquidos, espumas, geles) (Figura 2).

\section{Facilitadores de la higiene de manos}

\section{Infraestructura}

Una de las primeras medidas para favorecer la HM ha sido gestionar la fabricación de preparados de base alcohólica en aquellos países que no poseían esta fórmula, proporcionando la de la OMS para su elaboración; y mejorar su distribución ${ }^{6}$, con el fin de que el acceso del personal de salud al producto no fuese una limitación. En la misma línea se encuentra el estudio de Apisarnthanarak y cols., que se centró en aumentar la disponibilidad de los productos de base alcohólica en el punto de atención del paciente y en lugares de permanencia del personal como la estación de enfermería ${ }^{7,8}$.

Para reforzar la importancia que tiene la disponibilidad del producto para el personal, otro estudio describió el efecto de la localización del desinfectante de manos en el cumplimiento de $\mathrm{HM}^{8}$, tomando en cuenta la exclusividad de los dispensadores, que estos fuesen de un color diferente y que su ubicación fuera cerca del lavamanos o cercano a otros dispensadores como el del jabón.

Otro atributo de los preparados de base alcohólica es el ahorro de tiempo que conlleva la técnica de frotación (15-30 seg). Un estudio evaluó la acción bactericida en 10 seg. de contacto la cual logró una desinfección adecuada de las manos. Se combinan elementos que favorecen la utilización de estos productos de base alcohólica: no requiere infraestructura, el tiempo de contacto con las manos es reducido, pero a la vez, garantiza la eliminación de agentes de importancia epidemiológica, aumentando la adherencia de su uso? .

\section{Presentación del producto y disponibilidad}

En relación a la presentación del producto de base alcohólica, se evaluó la percepción del personal en tres presentaciones (líquido, espuma y gel). Dentro de los principales hallazgos, el personal se inclinó por el producto que utiliza frecuentemente.

Cuando se comparó entre las tres presentaciones, el gel es el preferido por su fácil aplicación, absorción, bajo nivel de residuos posterior a la frotación y secado rápido. La cantidad de producto se mencionó como un elemento importante; entre 1,5 y $0,7 \mathrm{ml}$ el personal tuvo mayor tolerabilidad ${ }^{10}$.

Cuando se evaluó la disponibilidad del producto, la difusión gráfica del alcohol gel, dispensadores con el producto y carteles alusivos a los 5 momentos de la HM, favoreció su uso ${ }^{11}$.

\section{Capacitación al personal de salud}

El personal del área de la salud tiene la percepción que el método de desinfección con productos de base alcohólica para la desinfección de las manos es más efectivo que la otra técnica (lavado de manos) para aumentar la adherencia a la $\mathrm{HM}^{12}$

Hay varios autores que proponen la capacitación al personal basada en aspectos teóricos y prácticos como una alternativa de solución para mejorar la adherencia ${ }^{1,5,8,12-14}$, junto con comprender la importancia de las IAAS.

Los programas educativos se basan en el comportamiento del personal (Banduras, 1982), utilizando como tema central el concepto de HM con preparados de base alcohólica en los 5 momentos de la atención. Los resultados reflejan un aumento en la adherencia, desde 54\% hasta $75 \%$ post intervención ${ }^{13}$. Al evaluar al personal de salud, este fue separado por estamento, destacando que el estamento médico aumentó su adherencia por sobre otros estamentos. Esto podría relacionarse a que su cumplimiento inicial fue el más bajo, por lo tanto, la estrategia de capacitación es importante en aumentar la adherencia a la $\mathrm{HM}^{14}$.

\section{Barreras para la higiene de manos}

\section{Infraestructura}

Disponer de recursos limitados para implementar dispensadores de alcohol gel en cada punto de atención es mencionado por el estudio patrocinado por la OMS, el cual incluyó países de bajos, medios y altos recursos ${ }^{6}$, ya que, la inversión inicial es significativa. 
Los dispensadores de preparados de base alcohólica se encontraron dentro de la habitación del paciente consignando como punto de atención, sin embargo, de acuerdo el modelo de los 5 momentos, el momento 1 y el 5 ocurren fuera de la habitación del paciente. En este estudio se evidenció que los dispensadores no se encontraban en todos los puntos recomendados ${ }^{8}$.

\section{Presentación del producto y disponibilidad}

La presentación en formato líquido no fue bien aceptada. El personal manifestó que fue difícil calcular la dosis a utilizar, no poseía buena absorción y fue de fácil derrame al piso en dosis mayores $(3 \mathrm{ml})$. Además se presentaron otros dos inconvenientes, entre más producto utilizado generó una falsa sensación de seguridad y requirió mayor tiempo de frotación para lograr su absorción ${ }^{10}$ y comparándolo con la presentación en gel, el formato líquido ocasionó mayor sequedad de la piel en las manos del personal e irritación si este fue utilizado en piel no sana.

Otra barrera que se consideró fue el tipo de dispensador. Si este no era fácil de utilizar y no entregaba la cantidad necesaria, el personal simplemente no acudió al desinfectante ${ }^{10}$.

\section{Capacitación}

Una de las limitaciones que manifestó el personal de salud fue no comprender los beneficios de usar productos de base alcohólica para la $\mathrm{HM}^{10}$.

Es importante tener en cuenta el nivel de conocimiento del personal de salud sobre la HM para dirigir las capacitaciones, sin embargo, según el estudio realizado por Kusain y cols. determinó que la retroalimentación regular no demostró ser una medida efectiva para incentivar al personal y aumentar la adherencia. Un alto nivel de conciencia no se encuentra necesariamente asociado con el comportamiento positivo. Que el personal se encuentre capacitado, no es sinónimo de que va adherir a la práctica de $\mathrm{HM}^{15}$.

\section{Discusión}

Esta revisión de la literatura especializada permitió conocer aquellos factores facilitadores y barreras frente al uso de preparados de base alcohólica con la finalidad de aumentar la adherencia a la HM, enmarcado en la estrate- gia multimodal de la OMS. Dentro de los facilitadores encontrados en esta revisión están: los atributos del producto de base alcohólica, el menor tiempo de aplicación ${ }^{12,14}$, la instalación de dispensadores en puntos estratégicos para su fácil acceso, por ende, mayor cumplimiento a la HM, y su localización o ubicación en el punto de atención ${ }^{5,7,14}$.

Contar con productos de base alcohólica accesibles logró desplazar el uso del agua y jabón, favoreciendo la técnica de frotación de manos y aumentando la adherencia $^{12}$. Otros atributos que se le confieren al alcohol gel como principal producto es que actúa más rápido, es eficaz en la disminución de microorganismos, posee una sensación de limpieza, no es pegajoso, y causa menos dermatitis, por lo que posee mejor tolerabilidad entre el personal de salud ${ }^{8,10}$

Un indicador que se relaciona con el aumento en la adherencia es el consumo del producto de base alcohólica medido en $\mathrm{ml}$. Posterior a una intervención hay en promedio un aumento de $2 \mathrm{ml}$ más que el consumo basal ${ }^{5,10,11,16}$.

La última etapa de la estrategia es establecer un clima de seguridad institucional ${ }^{1}$. Para lograr esto se propone incorporar el tema de la HM en actividades de capacitación, en reuniones clínicas, y de seguridad del paciente ${ }^{5}$.

Dentro de las barreras descritas para la adherencia a la HM se encuentran el número insuficiente de dispensadores del producto, el desconocimiento del personal en relación a la técnica de frotación y el comportamiento de no cambiar las prácticas ${ }^{17}$. Al ubicar juntos los dispensadores de los distintos productos para la HM (alcohol, jabón, crema hidratante) genera duda y confusión en qué producto utilizar. Como error frecuente aparece el uso de dos productos simultáneos como jabón y crema hidratante ${ }^{18}$. Otra barrera es la cantidad del producto, a mayor volumen $(3 \mathrm{ml})$ existe menos adhesión, porque la absorción se torna más lenta ${ }^{10}$.

Los facilitadores reconocidos deben potenciarse al momento de implementar el uso de productos de base alcohólica en los hospitales. Debe considerarse la elección del producto, la ubicación de los dispensadores en el punto de atención y la capacitación permanente sobre su uso, de acuerdo a los 5 momentos de la atención ${ }^{2}$.

Una de las limitaciones de esta revisión fue la ausencia de artículos provenientes de Latinoamérica, por lo tanto, se desconoce la implementación de la estrategia multimodal de la OMS para aumentar la adherencia de la HM en nuestra región. 


\section{Referencias bibliográficas}

1.- Guía de la OMS sobre la higiene de manos en la atención de la salud. OMS 2009. WHO/IER/ PSP/2009.07. Cap. 2.1. Disponible en: http:// cmas.siu.buap.mx/portal_pprd/work/sites/hup/ resources/LocalContent/247/2/guia_lavado_de manos.pdf. Fecha de acceso: 30 enero de 2019

2.- Guía de aplicación de la estrategia multimodal de la OMS para la mejora de la higiene de las manos. 2009. WHO/IER/ PSP/2009.02. Disponible en: https://apps. who.int/iris/bitstream/handle/10665/102536/ WHO IER PSP 2009.02 spa. pdf? sequence $=1 \&$ isAllowed=y. Fecha de acceso: 25 mayo 2019

3.- Medidas de prevención de la transmisión de microorganismos entre pacientes hospitalizados. Higiene de manos. Enferm Infecc Microbiol Clin. 2014; 32: 603-9. doi: 10.1016/j.eimc.2014.02.003

4.- Allegranzi B, Pittet D. Role of hand hygiene in healthcare-associated infection. J Hosp Infect. 2009; 73: 305- 15. doi:10.1016/j. jhin.2009.04.019

5.- Fariñas-Alvarez C, Portal-María T, FlorMoralesV, Aja-Herrero A, Fabo-Navarro M, Lanza-Marín S, et al. A multimodal strategy to improve adherence to hand hygiene in a university hospital. Rev Calid Asist. 2017; 32: 50- 56 [Internet] [Consultado 28 mayo 2019]. Disponible desde https://www.ncbi. nlm.nih.gov/pmc/articles/PMC5141532/. DOI:10.1016/j.cali.2016.06.011

6.- Allegranzi B, Gayet-Ageron A, Damari N,
Bengaly L, McLaws M L, Moro M L, et al. Global implementation of WHO's multimodal strategy for improvement of hand hygiene: a quasy-experimental study. Lancet Infect Dis. 2013; 13: 843-51 doi: 10.1016/ S14733099(13)70163-4.

7.- Cure L, Van Enk R. Effect of hand sanitizer location on hand hygiene compliance. Am J Infect Control 2015; 43: 917-21. doi: 10.1016/j. ajic.2015.05.013.

8.- Stackelroth J, Sinnott M, Shaban R. Hesitation and error: Does product placement in an emergency department influence hand hygiene performance?. Am J Infect Control 2015; 43: 913- 6. doi: 10.1016/j. ajic.2015.04.199

9.- Chojecka A, Tarka P, Kierzkowska A, Nitsch-Osuch A, Kanecki K. Neutralization efficiency of alcohol based products used for rapid hand disinfection. Rocz Panstw Zakl Hig. 2017; 68: 389- 94.

10.- Greenaway R E, Ormandy K, Fellows C, Hollowood T. Impact of hand sanitizer format (gel/foam/liquid) and dose amount on its sensory properties and acceptability for improving hand hygiene compliance. J Hosp Infect. 2018; 100: 195-201. doi: 10.1016/j. jhin.2018.07.011.

11.- Molina-Cabrillana J, Dorta-Hung M E, Otero Sanz L, Hernandez Vera J, MartínRodríguez M, García de Carlos P. Influence of promotional material on hand hygiene in the safety culture of a tertiary hospital. Rev Calid Asist. 2016; 31(S1): 55-61. doi: 10.1016/j. cali.2016.01.002.
12.- Monistrol O, Calbo E, Riera M, C Nicolás, Font $\mathrm{R}$, Freixas N, et al. Impact of a hand hygiene educational programme on hospital - acquired infections in medical wards. Clin Microbiol Infect. 2012; 18: 1212-8. doi: 10.1111/j.14690691.2011.03735.x.

13.- Teker B, Ogutlu A, Gozdas H.T, Ruayercan S, Hacialioglu G, Karabay O. Factors affecting hand hygiene adherence at a private hospital in Turkey. Eurasian J Med. 2015; 47: 208-12. doi: 10.5152/eurasianjmed.2015.78.

14.- Apisarnthanarak A, Eiamsitrakoon T, Mundy L. Behavior - based interventions to improve hand hygiene adherence among intensive care unit healthcare workers in Thailand. Infect Control Hosp Epidemiol. 2015; 36: 517- 21. doi:10.1017/ice.2015.1

15.- Amil Kusain Jr., Jeffrey O. Assessing healthcare associated infections and hand hygiene perceptions amongst healthcare professionals. Int J Caring Sci. 2015; 8: 108-14.

16.- Lai C C, Lu M C, Tang H J, Chen Y H, Wu Y H, Chiang H T, et al. Implementation of a national quality improvement program to enhance hand hygiene in nursing homes in Taiwan. J Microbiol Inmunol Infect. 2019; 52: 345-51. doi: 10.1016/j.jmii.2018.09.007.

17.- Kingston L, O'Connell N H, Dunne C P. Hand hygiene-related clinical trials reported since 2010: a systematic review. J Hosp Infect. 2016; 92: 309-20. doi: 10.1016/j.jhin.2015.11.012.

18.- Collins McLaughlin A, Walsh F. Self-reported reasons for hand hygiene in 3 groups of health care workers. Am J Infect. Control 2012; 40 : 653-8. doi: 10.1016/j.ajic.2011.08.014. 